Cómo citar este artículo en bibliografías / Referencia

X Soengas Pérez, AM López-Cepeda, J Sixto-García (2019): "Dieta mediática, hábitos de consumo de noticias y desinformación en los universitarios españoles". Revista Latina de Comunicación Social, 74, pp. 1056 a 1070.

http://www.revistalatinacs.org/074paper/1371/54es.html

DOI: 10.4185/RLCS-2019-1371-54

\title{
Dieta mediática, hábitos de consumo de noticias y desinformación en los universitarios españoles
}

\author{
Media diet, consumption habits and misinformation among \\ Spanish university students
}

Xosé Soengas-Pérez [CV] [] [ [ ] Facultad de Ciencias de la Comunicación. Universidad de Santiago de Compostela, USC, España xose.soengas@ usc.es

Ana María López-Cepeda [CV] [] [ [ G ] Facultad de Periodismo. Universidad de CastillaLa Mancha, UCLM, España ana.lopezcepeda@uclm.es

José Sixto-García [CV] [@] [ ] Facultad de Ciencias de la Comunicación. Universidad de Santiago de Compostela, USC, España jose.sixto@usc.es

\begin{abstract}
s
[ES] Introducción. En el escenario mediático actual han cambiado las formas y las posibilidades de informarse, y en esta investigación analizamos el consumo de noticias en los medios, en Internet y en las redes por estudiantes de Ciencias Sociales, diferenciando los que cursan Periodismo. Metodología. Seleccionamos 100 estudiantes de tercero y cuarto curso de cinco universidades públicas ubicadas en ciudades con características socioeconómicas diferentes para comprobar si el entorno influye en el comportamiento y en el consumo de información, y si se refleja en las rutinas, en el interés y en las preferencias. Resultados y conclusiones. La prensa, la radio y la televisión siguen perdiendo audiencia y la mayoría de los alumnos ya se informan a través de soportes digitales porque están desconectados de los escenarios clásicos. Gracias a la tecnología, existe una nueva forma de relacionarse con el espacio y de gestionar el tiempo, y esta circunstancia afecta a las formas de acceso, a los contenidos informativos y a las posibilidades de conocer lo que sucede en la actualidad.
\end{abstract}

[EN] Introduction. In the current media environment, news consumption has undergone radical changes in terms of possibilities and forms. This research article focuses on the analysis of news consumption in traditional media, on the Internet and in social media among social science students, and particularly among journalism students. Methods. Data were collected from a sample of 100 thirdyear and fourth-year students from five public universities located in cities with different socioeconomic profiles, to determine whether environment has an impact on students' behaviour and news 
consumption habits, and whether this influence is reflected on students' routines, interest and preferences. Results and conclusions. The press, radio and television continue losing audiences and most students now get the news from digital media platforms as they are already disconnected from traditional news media outlets. Technological developments have enabled new ways of dealing with space and managing time, which have an impact on the way people access information, on news content itself and on people's possibilities to learn about what happens around them.

\section{Keywords}

[ES] dieta mediática; jóvenes; medios de comunicación; redes sociales; desinformación.

[EN] media diet; young people; media; social networks; disinformation.

\section{Contents}

[ES] 1. Introducción. 1.1 La oferta informativa y la segmentación de las audiencias. 1.2 Nuevos escenarios mediáticos y nuevos contextos informativos. 2. Objetivos. 3. Objeto de estudio. 4. Hipótesis. 5. Metodología. 6. Resultados. 6.1 El consumo de información en los medios, en Internet y en las redes sociales. 6.2. Los medios y las redes más consultados. 6.3. Los temas que más interesan. 6.4. Hábitos de consumo en prensa, radio, televisión y redes sociales. 6.5. Contrastar la información, ampliar datos y conocer varios puntos de vista. 6.6. La credibilidad de los soportes. 6.7. Los resultados por titulaciones, por sexos y por universidades. 7. Discusión y conclusiones. 8. Referencias bibliográficas.

[EN] 1. Introduction. 1.1. News supply and audience segmentation. 1.2. New media environments and new information contexts. 2. Objectives. 3. Object of study. 4. Hypothesis. 5. Methods. 6. Results. 6.1. News consumption in traditional media, on the Internet and in social media. 6.2. Most consumed news media outlets and networks. 6.3. Most attractive news sections. 6.4. News consumption in the press, radio, television and social networks. 6.5. Fact-checking, expanding on details and ensuring plurality. 6.6. Credibility of platforms. 6.7. Results by career, sex and university. 7. Discussion and conclusions. 8. References.

Traducción de CA Martínez-Arcos (Doctor en Comunicación, Universidad de Londres)

\section{Introducción}

Las formas y las posibilidades de informarse han cambiado entre los jóvenes españoles (CaseroRipollés, 2012; López-Vidales, González-Aldea \& Medina-de-la-Viña, 2011), una tendencia generalizada como demuestran estudios realizados en diferentes países (Silveira \& Amaral, 2018; Vernier, Cárcamo \& Scheihing, 2018). La tecnología democratiza el acceso a los contenidos, cada vez más especializados, y genera nuevos escenarios informativos (Pérez-Escolar, 2016; Sánchez-Duarte et al., 2015), que implican nuevas formas de presentar los hechos a la audiencia, nuevas formas de distribuir las noticias y nuevas formas de consumir la información, más flexibles gracias a la sociedad multipantalla (Catalina-García, García-Jiménez \& Montes-Vozmediano, 2015; Pérez-Tornero, 2008; Velásquez et al., 2018).

En el escenario mediático actual los soportes tradicionales también tienen una versión digital y los contenidos informativos que antes sólo se publicaban en papel o se emitían por radio o por televisión, ahora pueden consultarse en Internet y en las redes sociales, en cualquier momento y desde cualquier lugar, de forma instantánea, tanto en directo como en diferido (García-Orosa \& López-García, 2016; 
Masip et al., 2015), sin estar condicionados por el horario fijo y predeterminado que caracterizaba las emisiones analógicas (Campos-Freire, 2015; Suárez-Villegas, 2015). De hecho, la mayoría de los jóvenes ya se informa a través de la Red (Casero-Ripollés, 2012) porque conviven en unos contextos que están muy desconectados de los escenarios clásicos y, tanto su dieta mediática como sus comportamientos, están condicionados por factores que no existían antes de la digitalización (DovalAvendaño, Domínguez-Quintas \& Dans-Álvarez-de-Sotomayor, 2018; García-García, GértudrixBarrio \& Gértudrix-Barrio, 2014; García-Jiménez, Tur-Viñes \& Pastor-Ruiz, 2018).

Los datos sobre el consumo de medios reflejan una caída progresiva en los lectores de prensa y en la audiencia de los informativos de radio y de televisión en los últimos años, como se puede comprobar en el cuadro número 1.

Cuadro 1. Penetración de los medios entre los jóvenes de 20 a 24 años

\begin{tabular}{|c|c|c|c|c|c|c|c|c|}
\hline \multicolumn{9}{|c|}{ Año 2017} \\
\hline & Población & Diarios & Suplementos & Revistas & Radio & TV & Cine & Internet \\
\hline Total & 39.783 & 24,3 & 8,7 & 32,8 & 59,3 & 85,2 & 3,9 & 75,7 \\
\hline 20 a 24 & 2.242 & 17,5 & 5,1 & 32 & 57,14 & 79,1 & 6,5 & 92,4 \\
\hline \multicolumn{9}{|c|}{ Año 2014} \\
\hline Total & 39.681 & 29,8 & 12,7 & 41 & 61,0 & 88,6 & 3,8 & 60,7 \\
\hline 20 a 24 & 2.355 & 25,9 & 8,4 & 46,3 & 63,2 & 84,3 & 6,5 & 88,2 \\
\hline \multicolumn{9}{|c|}{ Año 2009} \\
\hline Total & 39.462 & 39,8 & 21,9 & 51,3 & 55,3 & 89,0 & 4,3 & 34,3 \\
\hline 20 a 24 & 2.714 & 40,5 & 17,8 & 61,6 & 58,4 & 87,1 & 7,9 & 56,2 \\
\hline \multicolumn{9}{|c|}{ Año 2004} \\
\hline Total & 36.405 & 41,1 & 30,4 & 55,1 & 56,8 & 89,6 & 8,9 & 16,8 \\
\hline 20 a 24 & 2.987 & 44,2 & 28,6 & 71,1 & 63,7 & 87,4 & 21,5 & 31,7 \\
\hline \multicolumn{9}{|c|}{ Año 1999} \\
\hline Total & 34.497 & 35,2 & 31,3 & 53,3 & 53,0 & 89,4 & 10,2 & 7,0 \\
\hline 20 a 24 & 3.357 & 38,9 & 37,5 & 72,1 & 63,6 & 86,3 & 25,6 & 14,2 \\
\hline
\end{tabular}

Fuente: EGM: Marco general de los medios en España. Los datos de penetración reflejan el porcentaje de la audiencia en relación a la población, que se expresa en miles (000). Acceso: https://www.aimc.es/otrosestudios-trabajos/marcogeneral/descarga-marco-general/.

\subsection{La oferta informativa y la segmentación de las audiencias}

Los soportes clásicos no han adaptado su oferta a las exigencias de los diferentes sectores de la sociedad y los jóvenes muestran poco interés por los contenidos que ofrecen los medios tradicionales, porque no se corresponden con sus necesidades informativas. La información generalista que publican la mayoría de los medios de comunicación no encaja con la fragmentación y con la segmentación actual de las audiencias (Polo-López, Miotto \& Fondevila-Gascón, 2018; Rodríguez-Vázquez, DireitoRebollal \& Silva-Rodríguez, 2018; Santiago, 2017), cada vez más acostumbradas a los contenidos especializados, e incluso personalizados, que existen en Internet y en las redes sociales.

La caída de lectores de prensa y de las audiencias de los informativos de radio y de televisión es considerada por varios autores como un indicador que señala una falta de interés por parte de los jóvenes por lo que sucede en el mundo y una tendencia hacia la desinformación (Marchi, 2012), pero el consumo de medios tradicionales no se puede vincular con el grado de conocimiento de la actualidad, porque existen otras vías para informarse, como Internet y las redes sociales (GarcíaAvilés, Navarro-Maíllo \& Arias-Robles, 2014). Además, al hablar de desinformación (Rodríguez- 
Andrés, 2018), es necesario explicar en qué consiste estar informado (Goñi-Camejo, 2000; RíosOrtega, 2014), porque conocer todo lo que ocurre es imposible, y tampoco tienen las mismas necesidades, ni los mismos intereses informativos, todos los sectores de la población, ni todas las personas. Por eso, un hecho puede ser considerado importante o no según las circunstancias. En esta investigación abordamos la desinformación como una decisión voluntaria de alguien que no le interesa la actualidad. En cambio, descartamos como objeto de estudio la desinformación que se impone desde el poder. Aquí no analizamos el tratamiento informativo ni el enfoque de las noticias, ni la manipulación ni la censura que ejercen los medios de comunicación para ocultar una parte de la realidad, casi siempre por presiones políticas o económicas, procedentes del ámbito público o privado (Jacquard, 1988; Serrano, 2009).

Entre los diferentes teóricos e investigadores especialistas en desinformación, destaca Durandin (1995), quien deja claro que no existe un consenso para definir y establecer unos parámetros que permitan determinar en qué consiste estar informado, qué es necesario saber para estar informado o cuándo se considera que una persona está bien informada. Y también conviene diferenciar entre estar informado, ser experto y ser culto (Ribes-Iñesta, 2011). Además, es necesario distinguir entre información cualitativa, información cuantitativa e información útil, porque no somos capaces de gestionar todos los datos que recibimos a través de los medios, de Internet y de las redes sociales (Niño-González, Barquero-Cabrero \& García-García, 2017). Hay que establecer prioridades para hacer una selección adecuada de la oferta que está disponible en los diferentes soportes y plataformas, porque algunas informaciones tienen una vigencia o validez permanente y otras noticias tienen una caducidad o un valor puntual. Cada persona tiene unos intereses y unas necesidades específicas, y busca datos acordes con su perfil personal, social o laboral. Por eso es importante que la oferta informativa sea plural para que los ciudadanos puedan disponer de todo tipo de contenidos y seleccionen los que consideren adecuados (Humanes et al., 2013; Humanes \& Fernández-Alonso, 2015).

La saturación y el desinterés de la audiencia son dos problemas que afectan al escenario mediático actual y se pueden producir por un exceso de información o por la presencia de contenidos redundantes o poco útiles. Los criterios de los ciudadanos cambian porque la audiencia cada vez es más exigente y más crítica (Echegaray-Eizaguirre, 2015). Sus intereses y sus comportamientos evolucionan constantemente y ya no se conforman con las ofertas generalistas de la prensa, de la radio y de la televisión. Buscan contenidos alternativos y especializados, más acordes con sus inquietudes cotidianas y con su forma de vida. Los universitarios de hoy responden a un perfil muy diferente al que caracterizaba la generación de sus padres porque tienen unas necesidades y unos intereses distintos, que determinan el consumo de información en el contexto actual, tanto las temáticas como los soportes, e incluso los momentos y los lugares (Casero-Ripollés, 2012).

\subsection{Nuevos escenarios mediáticos y nuevos contextos informativos}

Los jóvenes forman parte de los nuevos públicos que configuran el escenario mediático, pero no son un sector de la población homogéneo, sino que pertenecen a diferentes extractos sociales y conviven en diferentes contextos, un factor que hay que tener en cuenta a la hora de analizar los posibles escenarios informativos y los distintos grados de interés por la actualidad. Cada grupo, incluso cada persona, tiene unas necesidades, tanto inmediatas como futuras, y unas preferencias, que son las que definen su perfil, porque a veces existe una correspondencia entre la ideología de los jóvenes y la línea editorial de los medios que consumen (Catalina-García, Montes-Vozmediano \& García-Jiménez, 2017). 
En el nuevo contexto informativo, caracterizado por una forma diferente de relacionarse con el espacio y de gestionar el tiempo, es necesario redefinir los conceptos de actualidad y de información útil. Con la actualización permanente de los contenidos en las versiones digitales de los medios y en las redes sociales, los hechos están sometidos a una revisión continua y a una caducidad casi inmediata. Al mismo tiempo, el exceso de datos y las posibilidades de acceso a las noticias de forma instantánea fomentan una lectura superficial de los temas, sin contrastarlos ni contextualizarlos, algo similar al periodismo de titulares (Mancinas-Chávez \& Moya-López, 2018).

\section{Objetivos}

El objetivo general de esta investigación es conocer cómo se informan los estudiantes españoles de Ciencias Sociales y, particularmente, los alumnos de Periodismo. Y los objetivos específicos consisten en averiguar qué soportes, qué medios y qué redes sociales consultan, en qué contexto, cuándo lo hacen, con qué frecuencia y qué contenidos les interesan más, para determinar cuáles son sus preferencias temáticas y sus hábitos de consumo informativo. Pero también nos interesa saber qué soporte les resulta más creíble, si consideran que la prensa, la radio, la televisión y las redes sociales ofrecen información útil, si amplían o contrastan algunos temas, con qué frecuencia lo hacen y si comentan la actualidad en general o algún tema en particular, con quién lo hacen y con qué frecuencia.

\section{Objeto de estudio}

Centramos nuestro objeto de estudio en el consumo de información en los medios de comunicación, en Internet y en las redes sociales por estudiantes de Ciencias Sociales, porque consideramos que se trata de un sector de la población con unos intereses académicos y profesionales que exigen conocer la actualidad. Al mismo tiempo, decidimos que la mitad de la muestra estuviera integrada por alumnos de Periodismo porque nos interesa saber qué relación tienen con los medios, con Internet y con las redes sociales los futuros informadores.

\section{Hipótesis}

Partimos de la siguiente hipótesis: los jóvenes universitarios españoles prefieren Internet y las redes sociales para informarse frente a los medios tradicionales. La prensa, la radio y la televisión disponen de versiones digitales, pero no han adaptado sus contenidos a las necesidades de una audiencia cada vez más fragmentada, más segmentada, más exigente y más crítica.

\section{Metodología}

Para realizar esta investigación utilizamos una metodología mixta, combinando aspectos cuantitativos con otros cualitativos. Así podemos conocer el grado de consumo de información en los medios tradicionales, en Internet y en las redes sociales, y también las razones por las que los jóvenes prefieren cada opción. Seleccionamos 100 estudiantes de tercero y cuarto curso de cinco universidades públicas ubicadas en ciudades con características socioeconómicas diferentes para comprobar si el entorno influye en el comportamiento y en el consumo de información, y si se refleja en las rutinas, en el interés y en las preferencias: Universitat Autònoma de Barcelona, Universidad Carlos III de Madrid, Universidad de Castilla-La Mancha, Universidad de Santiago de Compostela y Universidad de Sevilla, y trabajamos con 20 alumnos de cada una de las cinco universidades, 10 hombres y 10 mujeres. De los 100 alumnos seleccionados, 50 pertenecen a Periodismo y los otros 50 a otras titulaciones de Ciencias Sociales, que no consideramos necesario especificar cuáles son porque los datos de estos grados se analizan de forma conjunta. Sólo aislamos los resultados de los alumnos de Periodismo para 
realizar un análisis comparativo y comprobar si hay diferencias con la dieta mediática de los estudiantes de las otras titulaciones de Ciencias Sociales. Las encuestas se realizaron entre el 5 y el 15 de noviembre de 2018.

Las preguntas están pensadas para obtener un retrato de la dieta mediática de los estudiantes, de su interés por la actualidad y de su relación con los medios de comunicación, con Internet y con las redes sociales.

Las consultas se han hecho sobre los principales medios generalistas españoles y sobre las principales redes sociales: El País, El Mundo, ABC, Público, La Razón, RNE, SER, Onda Cero, COPE, TVE-1, TVE-La 2, TVE-Canal 24 horas, Antena 3, Cuatro, Telecinco, La Sexta, Facebook, Instagram, LinkedIn, YouTube, Twitter y Pinterest. Incluimos YouTube porque, aunque no es una red social al uso, ya que los usuarios no entran en ella para estar en contacto con sus amigos y compartir sus publicaciones, como hacen en Facebook o en Instagram, sí cumple las funciones sociales específicas que se le exigen a una red social, ya que permite conocer gente (finding people), estar en contacto con esa gente y con la que ya se conocía previamente (linking people), dejar comentarios, entablar conversación, mandar mensajes, seguir las publicaciones de otro usuario, conocer a miembros de la comunidad por recomendaciones, dar like a los vídeos o crear presencias específicas para las organizaciones (canales). En lo que difiere YouTube con respecto al resto de redes sociales es en el modelo de consumo, tanto en lo que respecta al producto (exclusivamente vídeo) como a la forma de consumirlo (se accede para consumir el producto y eso puede generar interacciones derivadas como en el resto de redes, pero el usuario ya conoce de antemano el producto o la temática que va a consumir) y de acceder a ese consumo (YouTube es la única red social que no exige registrarse y configurar un perfil personal para acceder a los contenidos) (Sixto, 2018).

\section{Resultados}

\subsection{El consumo de información en los medios, en Internet y en las redes sociales}

El 97\% de los estudiantes de Periodismo prefieren las redes sociales para informarse, en lugar de los medios clásicos, y el 92\% de los alumnos de otras titulaciones de Ciencias Sociales también señalan la misma opción. La prensa, con un $81 \%$ de lectores estudiantes de Periodismo y un $79 \%$ de Ciencias Sociales; la radio, con un $38 \%$ de oyentes de Periodismo y un $43 \%$ de Ciencias Sociales; y la televisión, con un $84 \%$ de espectadores de Periodismo y un $80 \%$ de Ciencias Sociales, ya no son los soportes más consultados por los universitarios. Estos datos confirman las tendencias recogidas en el EGM y en las investigaciones realizadas por Bárbara Yuste (2015), donde se demuestra cómo han cambiado los hábitos de consumo de información entre los jóvenes, abandonando progresivamente los medios convencionales para utilizar, cada vez más, las redes sociales y los dispositivos móviles.

Los porcentajes anteriores son altos, excepto en el caso de la radio, pero no se corresponden con un consumo de prensa y de televisión relevante de forma habitual, porque muchos estudiantes, como se puede comprobar a continuación, no se informan a diario a través de los medios.

Entre los alumnos que leen la prensa, el 15\% lo hace todos los días, el 6\% varias veces al día, el 37\% dos o tres veces a la semana, el $23 \%$ una vez a la semana, el $11 \%$ sólo cuando necesita documentarse sobre algún tema o en alguna circunstancia extraordinaria y el $8 \%$ nunca.

La radio es el medio menos utilizado por los estudiantes para informarse. El 9\% de los alumnos escucha informativos una vez al día, el 5\% varias veces al día, el 17\% dos o tres veces a la semana, el $48 \%$ una vez a la semana, el $10 \%$ cuando necesita documentarse sobre algún tema o en alguna circunstancia extraordinaria y el $11 \%$ nunca. 
En cambio, el consumo habitual de información en televisión es mucho mayor que el registrado en prensa y en radio. El $82 \%$ ve algún informativo una vez al día, el $9 \%$ varias veces al día, el $6 \%$ una vez a la semana y el $3 \%$ dos o tres veces a la semana.

Gráfico 1. Preferencias informativas de los alumnos por soportes

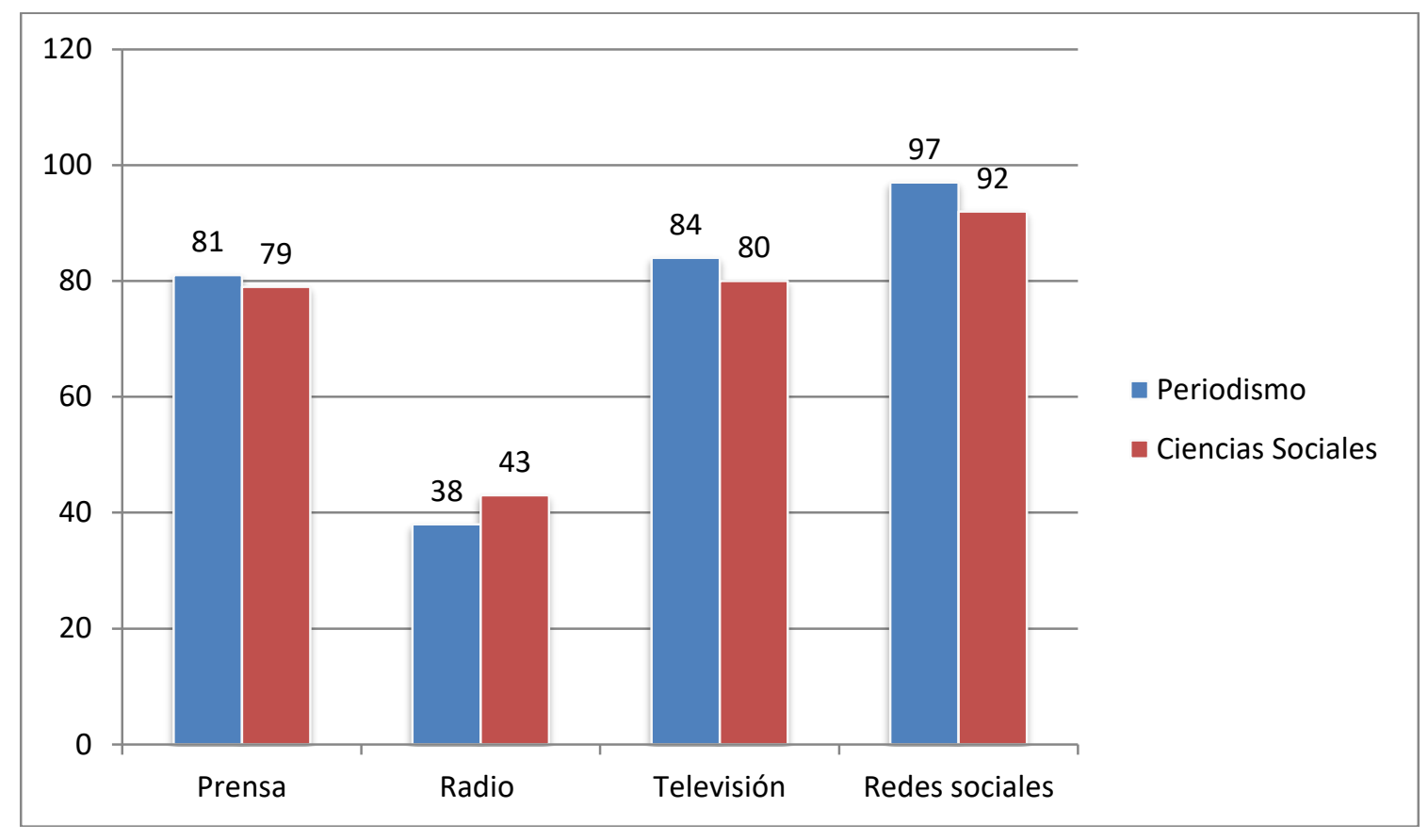

Fuente: elaboración propia

La actividad de los estudiantes en las redes sociales evidencia que este soporte digital es la opción preferida para informarse. El 5\% las consulta una vez al día, el 90\% varias veces al día, el 3\% cada dos o tres días y el $2 \%$ una vez a la semana. Aquí los porcentajes se invierten con respecto a la prensa, a la radio y a la televisión. Casi todos los alumnos consultan las redes sociales varias veces al día, una opción que en los medios tradicionales es minoritaria.

Internet es el responsable de muchos de los cambios que se han producido en el nuevo escenario mediático, pero aún no está consolidado como opción predominante para informarse en todos los soportes. El $98 \%$ de los universitarios prefiere las versiones digitales de los periódicos y el $77 \%$ también escucha la radio en páginas web, pero el $79 \%$ ven los informativos de televisión siempre en la opción analógica.

\subsection{Los medios y las redes más consultados}

El País, con el $42 \%$ de lectores, es el periódico más consultado, seguido de El Mundo (33\%), Abc (27\%), Público (23\%) y La Razón (18\%). En los informativos de radio destaca la SER con el 37\%, seguida de Onda Cero (28\%), RNE (25\%) y COPE (24\%), las tres últimas con unos porcentajes muy similares. El 46\% de los alumnos prefiere los informativos de Antena 3, el 41\% La Sexta, el 38\% Telecinco, el 29\% TVE-1, el 8\% Canal 24 horas de TVE y el 6\% Cuatro. En las redes sociales también hay un favorito. Twitter, con el $72 \%$, es la red más habitual para informarse, seguida de Instagram (68\%), YouTube (52\%), Facebook (45\%), LinkedIn (34\%) y Pinterest (21\%). Es importante hacer 
constar que la suma de los porcentajes referidos a cada soporte a veces sobrepasa el $100 \%$ porque algunos alumnos consultan varios medios.

\subsection{Los temas que más interesan}

La información nacional (91\%), la información política (67\%), las noticias de sociedad y las de cultura, ambas con el 64\%, y la información internacional (61\%), son los contenidos que más les interesan a los alumnos. El 42\%, también muestra preferencia por los deportes. La economía (21\%) y la información institucional (19\%) son los temas menos consultados.

Gráfico 2. Preferencias informativas por temáticas

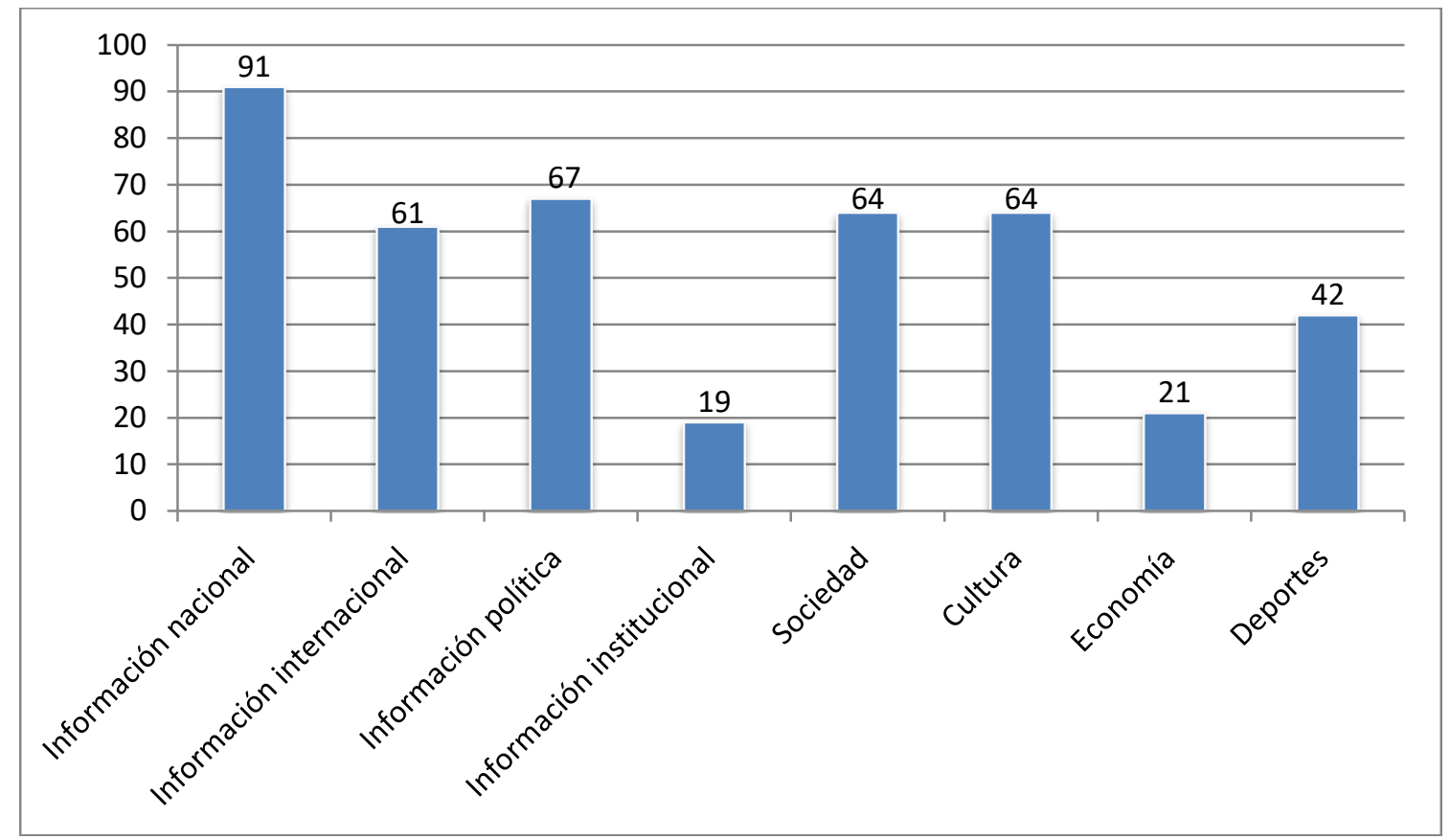

Fuente: elaboración propia

\subsection{Hábitos de consumo en prensa, radio, televisión y redes sociales}

Las versiones digitales de los medios han cambiado los hábitos de consumo de la información en los ciudadanos, y los estudiantes no son una excepción. Gracias al proceso de hibridación de medios la prensa, la radio y la televisión han integrado características que eran propias y específicas de otros soportes, y ese proceso ha modificado los protocolos para acceder a las noticias. Las diferentes franjas pautadas en esta investigación no son excluyentes porque un alumno puede consultar un medio varias veces al día, por eso la suma de los porcentajes parciales puede superar el $100 \%$.

El 9\% lee la prensa por la mañana para informarse antes de salir de casa, el $11 \%$ en el viaje hacia la universidad, el $15 \%$ en los descansos de clase, el $32 \%$ a mediodía, el $41 \%$ por la tarde y el $7 \%$ por la noche. El 10\% también escuchan la radio por la mañana, el $4 \%$ en el viaje hacia la universidad, el $3 \%$ en los descansos de clase, el $29 \%$ a mediodía, el $8 \%$ por la tarde y el $35 \%$ por la noche. El $6 \%$ ve los informativos de televisión por la mañana, ninguno reconoce que los ve en el viaje hacia la universidad, 
ni en los descansos de clase, aunque la tecnología lo permite, el 32\% a mediodía, el 3\% por la tarde y el $30 \%$ por la noche.

La actividad en las redes sociales es muy diferente y muy superior a la registrada en los demás soportes. El $62 \%$ consulta las redes sociales por la mañana antes de salir de casa, el $29 \%$ de camino a la universidad, el $71 \%$ en los descansos de clase, el $22 \%$ mientras come a mediodía, el $34 \%$ por la tarde y el $82 \%$ por la noche. Los datos reflejan que la mayoría de los alumnos consultan las redes sociales durante todo el día. Esta es la principal diferencia con respecto a los medios tradicionales (prensa, radio y televisión), que son consultados en momentos más concretos y en un porcentaje mucho menor.

\subsection{Contrastar la información, ampliar datos y conocer varios puntos de vista}

El 5\% de los alumnos lee más de tres periódicos habitualmente, el 7\% tres, el 18\% dos y el 70\% lee uno. Entre los oyentes de radio, el 3\% escucha más de tres emisoras, el 5\% tres, el 11\% dos y el $81 \%$ una. El 9\% ve más de tres informativos en televisión, el 17\% tres, el 45\% dos, y el 29\% uno. El 15\% consulta más de tres redes sociales, el $71 \%$ tres, el $11 \%$ dos y el 3\% una. Los datos demuestran que el consumo de información en televisión es más plural que en la prensa y en la radio, y la actividad en las redes sociales es mucho mayor que la registrada en los medios de comunicación tradicionales.

El 74\% de los estudiantes que consultan varios medios o varias redes sociales sólo consumen información para saber qué sucede en su entorno y en el mundo, pero no cuestionan el tratamiento informativo de las noticias. No tienen una actitud crítica frente al enfoque de los hechos, ni muestran interés por conocer puntos de vista alternativos. Estos datos contradicen la teoría que sostiene que las audiencias jóvenes son cada vez más críticas y más exigentes. Además, hay que tener en cuenta que leer varios periódicos, escuchar varios informativos de radio, ver varios telediarios o acceder a varias páginas web o a varias redes sociales no equivale necesariamente a contrastar una información porque en cada medio se pueden consultar contenidos diferentes. Sólo el $26 \%$ contrasta las noticias o amplía detalles para tener una versión más completa. Y de este 26\%, el 95\% lo hace de forma esporádica cuando le interesa algún tema y el 5\% de forma habitual. Las noticias falsas y la desinformación sólo le preocupan al 56\%. Además, el $41 \%$ le encuentra poca utilidad a la información que ofrecen la prensa, la radio y la televisión en sus versiones analógicas, y al 32\% tampoco le convencen los contenidos de las versiones digitales.

El 65\% no hace valoraciones de los acontecimientos que conoce. Únicamente el 35\% comparte sus opiniones o puntos de vista sobre el enfoque o sobre el tratamiento informativo de algunos temas que considera relevantes. De este 35\%, el 53\% lo hace con compañeros, el $40 \%$ con amigos, el 3\% con la familia y el $4 \%$ con profesores. Este último caso sólo se valora cuando el alumno intercambia opiniones sobre una noticia con un profesor voluntariamente, sin que forme parte de las actividades docentes universitarias.

\subsection{La credibilidad de los soportes}

La radio es el soporte con menos audiencia, pero es el más creíble para el 38\%, para el 25\% lo es la prensa y para el $20 \%$ la televisión. Las redes sociales ocupan el último lugar en fiabilidad (17\%), aunque la mayoría de los estudiantes se informan a través de ellas, concretamente el 97\% de alumnos de Periodismo y $92 \%$ de las otras titulaciones analizadas. 
Gráfico 3. Soporte que ofrece mayor nivel de credibilidad para los alumnos

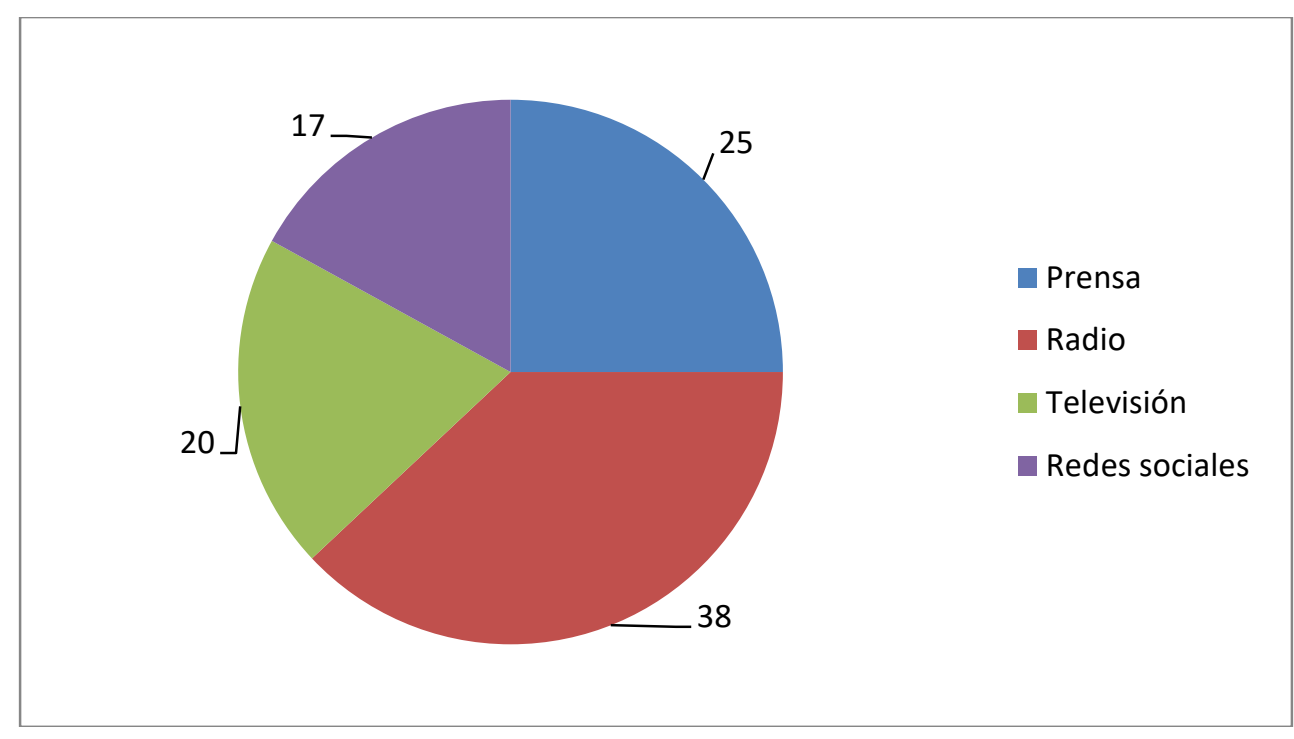

Fuente: elaboración propia

\subsection{Los resultados por titulaciones, por sexos y por universidades}

Los alumnos de Periodismo prefieren El País (41\%), la SER (52\%), La Sexta (35\%), Twitter (34\%) y la información nacional (29\%), y los estudiantes de las demás titulaciones de Ciencias Sociales prefieren El Mundo (36\%), Onda Cero (38\%), Antena 3 (32\%), Twitter (31) y la información internacional $(25 \%)$.

Los alumnos prefieren El País (56\%), la SER (50\%), Antena 3 (41\%), Twitter (43\%) y la información internacional (37\%), y las alumnas El Mundo (45\%), La SER (38\%), La Sexta (32\%), Instagram (29\%) y la información nacional (25\%).

Los datos de las universidades analizadas no presentan diferencias significativas entre ellas, lo cual demuestra que el entorno socioeconómico en el que están ubicados los centros de estudio no influye en las preferencias informativas.

\section{Discusión y conclusiones}

Los patrones de consumo de noticias han cambiado de forma significativa en los últimos años. Los resultados de esta investigación coinciden con la tendencia que reflejan los datos de audiencia del EGM, donde se puede comprobar que existe un abandono progresivo de los medios clásicos y un auge del consumo de información en las redes sociales, que han desplazado a la prensa, a la radio y a la televisión como referencia informativa para los jóvenes, especialmente los que tienen entre 20 y 24 años.

Ahora existen nuevos escenarios informativos, caracterizados por la fragmentación y por la segmentación de las audiencias, cada vez más especializadas, más exigentes y más críticas. Y en este contexto es donde hay que situar a los universitarios como parte de un público que tiene nuevas necesidades y que no se conforma con los contenidos que ofrecen los medios, tanto en los soportes 
tradicionales como en las versiones digitales, porque no han sabido adaptarse al nuevo escenario para competir con las redes sociales, ni aprovechar todas las oportunidades que ofrece la tecnología.

Gracias a Internet y a las redes sociales, los jóvenes disponen de una nueva forma de relacionarse con el espacio y de gestionar el tiempo, y esta circunstancia afecta a las formas de acceso a los contenidos y a las posibilidades de consumo de información. La tecnología permite consultar cualquier noticia, gratis, en cualquier momento, desde cualquier lugar, en directo o en diferido. Pero estas facilidades para acceder a todo tipo de contenidos no se corresponden siempre con un consumo relevante de información por parte de los universitarios, por lo menos al nivel que cabría esperar en un público cuyos intereses, inmediatos y futuros, están directamente vinculados al conocimiento y al análisis de la actualidad, especialmente en el caso de los estudiantes de Periodismo.

Es importante diferenciar los valores cuantitativos de los aspectos cualitativos. Los datos genéricos sobre consumo de información en los soportes clásicos no reflejan la situación real. En las cifras globales consta que el $80 \%$ lee la prensa, el $42 \%$ escucha la radio y el $82 \%$ ve la televisión, pero el $60 \%$ sólo se informa a través de un medio y el $25 \%$ sólo lo hace una vez a la semana. Los alumnos que leen varios periódicos, que sintonizan varias emisoras de radio o que ven informativos en varias cadenas de televisión cada día son pocos (8\%). Y todavía son menos los que consumen regularmente prensa, radio y televisión (6\%). Pero también hay que tener en cuenta que algunos alumnos se informan sólo a través de un medio de comunicación por afinidad, porque existen investigaciones como las de Catalina-García, Montes-Vozmediano y García-Jiménez (2017) que demuestran que muchos ciudadanos son fieles a los medios de comunicación cuya línea editorial concuerda con su ideología.

El nuevo escenario es complejo, incluso contradictorio. La oferta informativa es cada vez mayor, pero el $65 \%$ reconoce que hay saturación, redundancia y duplicidad en los contenidos. Y para el 53\% hay una mala gestión y una mala ordenación del mapa mediático, porque el número de medios que existe en España en estos momentos no se corresponde con una opción informativa atractiva, innovadora y diversa, con propuestas específicas de calidad y plurales para los distintos sectores de la población. Para el 59\% el mayor problema es que los medios siguen inmersos en la información generalista, cuando la audiencia actual busca contenidos concretos, acordes con sus necesidades y con sus intereses específicos. Además, consideran que es necesario redefinir los conceptos de actualidad y de información útil, para adaptarlos a la nueva realidad informativa.

Los datos demuestran que una parte importante de los universitarios no se informan con rigor. El 74\% no contrastan las noticias ni amplían la información, le dan credibilidad a los medios, se conforman una sola versión de los hechos y no se preocupan por conocer más puntos de vista sobre las noticias. Y tampoco aprovechan los espacios de convivencia virtual que facilita la tecnología para intercambiar opiniones, ni para analizar la actualidad. El $44 \%$ tampoco están preocupados por las noticias falsas, ni por la desinformación.

El 98\% de los universitarios prefiere las versiones digitales de los periódicos y el 77\% escucha la radio en páginas web, pero el $79 \%$ de los espectadores ven la televisión en la opción analógica y sólo el $21 \%$ ve los informativos en páginas web. En cambio, no se observan diferencias significativas en los hábitos de consumo y en la dieta mediática entre las diferentes universidades. El entorno socioeconómico en el que están ubicados los centros de estudio no influye en los resultados, pero sí tienen preferencias distintas los alumnos de Periodismo y los estudiantes de las demás titulaciones de Ciencias Sociales, y también los alumnos y las alumnas, tal como consta de forma detallada en el análisis de datos. 
La radio, el soporte con menos audiencia, es el más creíble y las redes sociales, las más consultadas, son las que tienen menos fiabilidad entre los universitarios.

- Este artículo forma parte de las actividades de dos proyectos de investigación. (RTI2018-096065-B-I00) del Programa Estatal de I+D+I orientado a los Retos de la Sociedad del Ministerio de Ciencia, Innovación y Universidades y del Fondo Europeo de Desarrollo Regional (FEDER) sobre "Nuevos valores, gobernanza, financiación y servicios audiovisuales públicos para la sociedad de Internet: contrastes europeos y españoles" "Usos y preferencias informativas en el nuevo mapa de medios en España: modelos de periodismo para dispositivos móviles" (Referencia: CSO2015-64662-C4-4-R), del Ministerio de Economía y Competitividad. Este proyecto está cofinanciado por el fondo estructural FEDER.

\section{Referencias bibliográficas}

F Campos-Freire (2015): “Adaptación de los medios tradicionales a la innovación de los metamedios". El profesional de la información, 24(4), pp. 441-450. DOI: 10.3145/epi.2015.jul.11

A Casero-Ripollés (2012): "Más allá de los diarios: El consumo de noticias de los jóvenes en la era digital”. Comunicar, 20(39), pp. 151-158. DOI: 10.3916/C39-2012-03-05

B Catalina-García, A García-Jiménez \& M Montes-Vozmediano (2015): “Jóvenes y consumo de noticias a través de Internet y los medios sociales". Historia y Comunicación Social, 20(2), pp. 601619. DOI: $10.5209 /$ rev_HICS. 2015.v20. n2.51402

B Catalina-García, M Montes-Vozmediano \& A García-Jiménez (2017): "Los jóvenes universitarios y sus pautas de consumo y difusión de noticias según la tendencia ideológica”, Fonseca, Journal of Communication, 15, pp. 57-73. DOI: 10.14201/fjc2017155773

M Doval-Avendaño, S Domínguez-Quintas \& I Dans-Álvarez-de-Sotomayor (2018): “El uso ritual de las pantallas en jóvenes universitarios/as. Una experiencia de dieta digital". Prisma Social, 21, pp. 481-499. Recuperado de http://revistaprismasocial.es/article/view/2323

G Durandin (1995): La información, la desinformación y la realidad. Barcelona: Paidós Ibérica.

L Echegaray-Eizaguirre (2015): "Los nuevos roles del usuario: audiencia en el entorno comunicacional de las redes sociales”. En Quintas-Froufe \& González-Neira (coords.), La participación de la audiencia en la televisión: de la audiencia activa a la social (pp. 27-46). Madrid: Asociación para la Investigación de Medios de Comunicación (AIMC).

JA García-Avilés, F Navarro-Maíllo \& F Arias-Robles (2014). "La credibilidad de los contenidos informativos en internet para los 'nativos digitales': estudio de caso". Palabra Clave, 17(3), pp. 875894. DOI: $10.5294 /$ pacla.2014.17.3.13

F García-García, M Gértudrix-Barrio \& F Gértudrix-Barrio (2014): “Análisis de la incidencia de la dieta de servicios digitales en la utilidad y confianza de la información en internet en los jóvenes universitarios". Communication \& Society, 27(1), pp. 59-81. 
A García-Jiménez, V Tur-Viñes \& Y Pastor-Ruiz (2018): “Consumo mediático de adolescentes y jóvenes. Noticias, contenidos audiovisuales y medición de audiencias”. Icono 14, 16(1), pp. 22-46. DOI: $10.7195 /$ ri14.v16i1.1101

B García-Orosa \& X López-García (2016): "Las redes sociales como herramienta de distribución online de la oferta informativa en los medios de España y Portugal". Zer, Revista de Estudios de Comunicación, 21(40), pp. 125-139. DOI: 10.1387/zer.16416

I Goñi-Camejo (2000): “Algunas reflexiones sobre el concepto de información y sus implicaciones para el desarrollo de las ciencias de la información”, ACIMED, 8(3), pp. 201-207. Recuperado de http://scielo.sld.cu/scielo.php?script=sci_arttext\&pid=S1024-

$94352000000300005 \& \operatorname{lng}=$ es\&nrm=iso

ML Humanes \& I Fernández-Alonso (2015): "Pluralismo informativo y medios públicos. La involución de TVE en el contexto del cambio político (2012-2013)”. Revista Latina de Comunicación Social, 70, pp. 270 a 287. Recuperado de http://www.revistalatinacs.org/070/paper/1046fu/16es.html DOI: 10.4185/RLCS-2015-1046

ML Humanes, MD Montero-Sánchez, R Molina-de-Dios \& A López-Berini (2013): "Pluralismo y paralelismo político en la información televisiva en España". Revista Latina de Comunicación Social, 68, pp. 566-581. Recuperado de http://www.revistalatinacs.org/068/paper/990_Fuenlabrada/24_Humanes.html DOI: 10.4185/RLCS2013-990

R Jacquard (1988): La desinformación: una manipulación del poder. Madrid: Espasa Calpe.

N López-Vidales, P González-Aldea \& E Medina-de-la-Viña (2011): “Jóvenes y televisión en 2010: Un cambio de hábitos". Zer, Revista de Estudios de Comunicación, 16(30), pp. 97-113. Recuperado de http://www.ehu.eus/ojs/index.php/Zer/article/view/4793/4657

R Mancinas-Chávez \& D Moya-López (2018): "Lucha a muerte por un click. La adaptación de los contenidos informativos a las necesidades de financiación en prensa digital". En Nogales-Bocio, Solans-García \& Marta-Lazo (eds.), Estándares e indicadores para la calidad informativa en los medios digitales (pp. 17-32). Sevilla: Ediciones Egregius.

R Marchi (2012): "With Facebook, blogs, and fake news, teens reject journalistic 'objectivity'". Journal of Communication Inquiry, 36(3), pp. 246-262. DOI: 10.1177/0196859912458700

P Masip, J Guallar, J Suau, C Ruiz-Caballero \& M Peralta (2015): "News and social networks: Audience behavior". El profesional de la información, 24(4), pp. 363 - 370. DOI:

10.3145/epi.2015.jul.02

JI Niño-González, M Barquero-Cabrero \& E García-García (2017): “Opinión pública e infoxicación en las redes: los fundamentos de la post-verdad". Vivat Academia, Revista de Comunicación, 139, pp. 83-94. DOI: $10.15178 /$ va.2017.139.83-94

M Pérez-Escolar (2016): "El cambio ecológico de la comunicación do it yourself. Transformación del escenario mediático tradicional desde la perspectiva del prosumidor”. En Mancinas-Chávez 
(coord.), Actas del I Congreso Internacional Comunicación y Pensamiento. Comunicracia y desarrollo social (pp. 19-34). Sevilla: Egregius.

JM Pérez-Tornero (2008): "La sociedad multipantallas: retos para la alfabetización mediática". Comunicar, 26(31), pp.15-25. DOI: 10.3916/c31-2008-01-002

M Polo-López, G Miotto \& JF Fondevila-Gascón (2018): "My Time: Incidencia de la televisión a la carta en la evolución del prime time en España”. Revista Latina de Comunicación Social, 73, pp. 208-227. Recuperado de http://www.revistalatinacs.org/073paper/1253/12es.html DOI: $10.4185 / R L C S-2018-1253$

E Ribes-Iñesta (2011): "El concepto de competencia: su pertinencia en el desarrollo psicológico y la educación”. Bordón, 63(1) pp. 33-45.

J Ríos-Ortega (2014): "The concept of information in Library Science, Sociology and Cognitive Science”, Investigación Bibliotecológica, 28(62) pp. 143-179.

R Rodríguez-Andrés (2018): "Fundamentos del concepto de desinformación como práctica manipuladora en la comunicación política y las relaciones internacionales". Historia y comunicación social, 23(1) pp. 231-244. DOI: 10.5209/HICS.59843

AI Rodríguez-Vázquez, S Direito-Rebollal \& A Silva-Rodríguez (2018): “Audiencias crossmedia: nuevas métricas y perfiles profesionales en los medios españoles". El profesional de la información, 27(4) pp. 793-800. DOI: 10.3145/epi.2018.jul.08

JM Sánchez-Duarte, MV Bolaños-Huertas, R Magallón-Rosa \& V Anderica-Caffarena (2015): “El papel de las tecnologías cívicas en la redefinición de la esfera pública". Historia y Comunicación Social, 20(2), pp. 483-498. DOI: 10.5209/rev HICS. 2015.v20. n2.51396

F Santiago (2017): Fragmentación de la audiencia. Retos de la medición. Barcelona: Asociación para la Investigación de Medios de Comunicación, AIMC. Recuperado de https://goo.gl/T7Xcgg

P Serrano (2009): Desinformación: cómo los medios ocultan el mundo. Barcelona: Editorial Península.

P Silveira \& I Amaral (2018): “Jovens e práticas de acesso e de consumo de notícias nos media sociais", Estudos em Comunicaçao, 1(26), pp. 261-280. DOI: 10.20287/ec. n26.v1.a15

J Sixto (2018): Gestión profesional de redes sociales. Rutinas y estrategias de Community Management. Salamanca: Comunicación Social.

JC Suárez-Villegas (2015): "Nuevas tecnologías y deontología periodística: comparación entre medios tradicionales y nativos digitales". El profesional de la información, 24(4) pp. 390-395. DOI: 10.3145/epi.2015.jul.05

A Velásquez, D. Renó, AM Beltrán-Flandoli, JC Maldonado-Vivanco \& C Ortiz León (2018): “De los mass media a los medios sociales: reflexiones sobre la nueva ecología de los medios". Revista Latina de Comunicación Social, 73, pp. 583-594. Recuperado de 
http://www.revistalatinacs.org/073paper/1270/29es.html

DOI: $10.4185 / R L C S-2018-1270$

M Vernier, L Cárcamo \& E Scheihing (2018): "Pensamiento crítico de los jóvenes ciudadanos frente a las noticias en Chile", Comunicar, 26(54), pp. 101-110. DOI: 10.3916/C54-2018-10

B Yuste (2015): "Las nuevas formas de consumir información de los jóvenes". Revista de Estudios de Juventud, 108, pp. 179-191.

\section{Cómo citar este artículo en bibliografias / Referencia}

X Soengas-Pérez, AM López-Cepeda, J Sixto-García (2019): "Dieta mediática, hábitos de consumo de noticias y desinformación en los universitarios españoles". Revista Latina de Comunicación Social, 74, pp. 1056 a 1070. http://www.revistalatinacs.org/074paper/1371/54es.html

DOI: $10.4185 / R L C S-2019-1371-54$

- En el interior de un texto:

X Soengas-Pérez, AM López-Cepeda, J Sixto-García (2019: 1056 a 1070) ...

$\mathrm{o}$

...X Soengas-Pérez et al, 2019 (1056 a 1070) ...

Artículo recibido el 7 de diciembre Aceptado el 10 de junio.

Publicado el 12 de junio de 2019 